\title{
Implementation of Markov Chain for Advanced Communication in MIMO-OFDM
}

\author{
Gauri B. Maske', Prof. S. B. Mule ${ }^{2}$ \\ E\&TC Department, Sinhgad College of Engineering, Vadgaon (BK), Pune ${ }^{1,2}$
}

\begin{abstract}
As per increasing demand of higher data rate with error free communication network, a combination of MIMO-OFDM is used. To fulfill the demands of separate and independent data transmission a new technique called Markov Chain is proposed. Since, Markov Chain uses independent channels there are less possibilities of Inter Channel Interference (ICI) and Inter Symbol Interference (ISI). Different fading channels are considered for BER calculation. Error rate calculation helps user to decide the efficient channel for data transmission. This will result into flexible and user friendly communication network.
\end{abstract}

Keywords: MIMO, OFDM, Markov Chain, BER, MIMO-OFDM.

\section{INTRODUCTION}

In late 1970s, communication is carried out through wired transmission. Further evolution of communication network is transformation from one technology to another. Technology evolution in communication network is $1 \mathrm{G}, 2 \mathrm{G}, 3 \mathrm{G}, 4 \mathrm{G}$ LTE and now working on 5G. These technologies work on different techniques such as first two generations i.e. $1 \mathrm{G}$ and $2 \mathrm{G}$ uses circuit switching, spectrum between $2 \mathrm{G}$ and $\mathrm{G}$ uses both circuit and packet switching. Further technologies, including 5G work on packet switching. On the basis of licensed and unlicensed spectrum, all evolved generations uses licensed spectrum whereas technologies like Bluetooth, WI-Max and Wi-Fi uses unlicensed spectrum.

\section{Evolution of technologies:}

\section{$1 \mathrm{G}$ technology:}

In 1980s, communication through $1 \mathrm{G}$ is in analog form over channel capacity of $30 \mathrm{KHz}$ and frequency band of $824-$ 894MHz. There are several drawbacks like low capacity, unreliable hand off, and low voice quality. It uses only one channel for transmission. These drawbacks are overcome in future technologies.

\section{G technology:}

In 1990s, 2G system is introduced with use of digital system. It uses services like SMS, MMS over frequency band of $1.8 \mathrm{GHz}$ and bandwidth of $900 \mathrm{MHz}$. In $2 \mathrm{G}$, it is difficult to handle services like it video.

\section{G technology:}

$3 \mathrm{G}$ technology is introduced in 2000 with the combination of high data rate with Internet Protocol (IP). 3G technologies uses 1.6-2 GHZ frequency band over bandwidth of $100 \mathrm{MHz}$. Limitation of $3 \mathrm{G}$ networks are higher bandwidth and higher cost and size of $3 \mathrm{G}$ phones.

\section{G technology:}

In $2010,4 \mathrm{G}$ system with high speed of $100 \mathrm{Mbps}-1 \mathrm{Gbps}$ is followed by users. $4 \mathrm{G}$ technologies has advantages that it uses LTE and Wi-Max technologies. Devices with $4 \mathrm{G}$ technologies can be used anywhere and anytime. With these advantages, $4 \mathrm{G}$ system has disadvantages like low battery life, difficult hardware implementation.

\section{G technology:}

5G technology uses OFDM technique. It would be called as wwww(World Wide Wireless Web) coz it has no limitations and is made user friendly. 5G technology works in physical and data link layer which indicates 5G technology as Open Wireless Architecture (OWA). An idea to shift towards 5G is to overcome the drawbacks of 4G technology and develop a flexible and secure network.

With the considerations of users demand, MIMO-OFDM technique is carried out to fulfill demands. MIMO system with multiple active antennas at transmitter and receiver satisfies the demand of higher data rate wireless communication network. To make system user friendly, 5G network with additional benefits than other network 
Vol. 5, Issue 7, July 2017

technologies is taken into considerations. Combination of MIMO-OFDM-MARKOV helps researchers to work on a flexible and user friendly communication network. The main aim of designing this system is to obtain communication with less BER. Secured communication is the main purpose behind this implementation. Fading channels like Rayleigh, Rician with AWGN are used.

\section{LITERATURE REVIEW}

Effective implementation of a system requires detailed study and analysis of various papers.

In [1], OFDM-MIMO with index modulation is considered to boost the data rate. It uses separate data frames for demodulation at receiver. Due to different error performances it is difficult to analyze the number of detectors. In [2], a new clean approach of $5 \mathrm{G}$ is proposed for radio access technology which is based on TDD system. For flexible UL/DL, OFDM is used which results into extension of MIMO. For an integration of heterogeneous IPv6, Ethernet-over-Radio link is used. With the focus on flexibility, automatic organization of network combination of Ultra Dense Network with $\mathrm{C}$-Ran is proposed in [3]. The use of C-RAN results into advantageous high data rate at low traffic. With the scheduling period of $1 \mathrm{~ms}$, [4] proposes a resource scheduling which obtains high throughput. As compared to conventional scheme, to increase the throughput combination of implementation scheme and search scheme is developed. In communication network, after channel classification characteristics of channel can be estimated using Markov chain. To obtain significant result with minimum errors Markov chain is used over several channels [5].

\section{DESCRIPTION OF MIMO-OFDM}

MIMO-OFDM combines two techniques i.e. MIMO system which transmits signal over multiple antennas to multiply the transmission capacity. Other technique is OFDM, in which signals are orthogonally divided into large number of sub-channels. Orthogonally arrangement helps to reduce the Inter Channel Interference. Combination of MIMO system with OFDM works powerfully in communication network. MIMO system helps to reduce the multipath propagation while OFDM system helps to reduce interference.

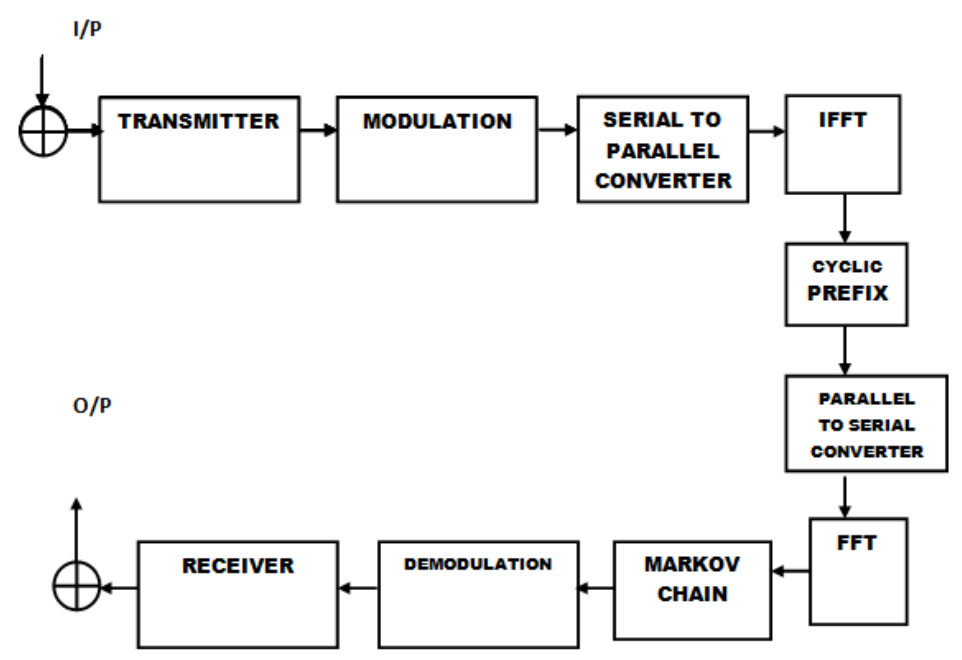

Fig.1 Proposed block diagram of a system

As per above fig.1, Markov chain is a stochastic process. It refers to a sequence of variables that moves independently. Changes in states are referred as transitions. Random change in system results in unpredictable certainty of states in Markov chain. Due to independently working of variables, it is possible to reduce the effects of noise in channel.

\section{RESULTS AND DISCUSSION}

With the considerations of BER calculation, it is expected that the communication through $5 \mathrm{G}$ technology is much better than the other technologies. Referring to the simulation results BER for different values shows that use of different channels will results into different error rate. The proposed communication network uses Markov chain for better communication. As per figure. 2 BER in Rayleigh channel is shown. Fig. 3 and fig.4 shows the BER calculation with $K=2 \mathrm{~dB}$ and $\mathrm{K}=15 \mathrm{~dB}$ respectively. From these graphs it can be determined that with more $\mathrm{K}$ values, we can get less BER. Less BER helps to select better communication channel. 
UGC Approved Journal

IJIREEICE

International Journal of Innovative Research in Electrical, Electronics, Instrumentation and Control Engineering

ISO 3297:2007 Certified

Vol. 5, Issue 7, July 2017

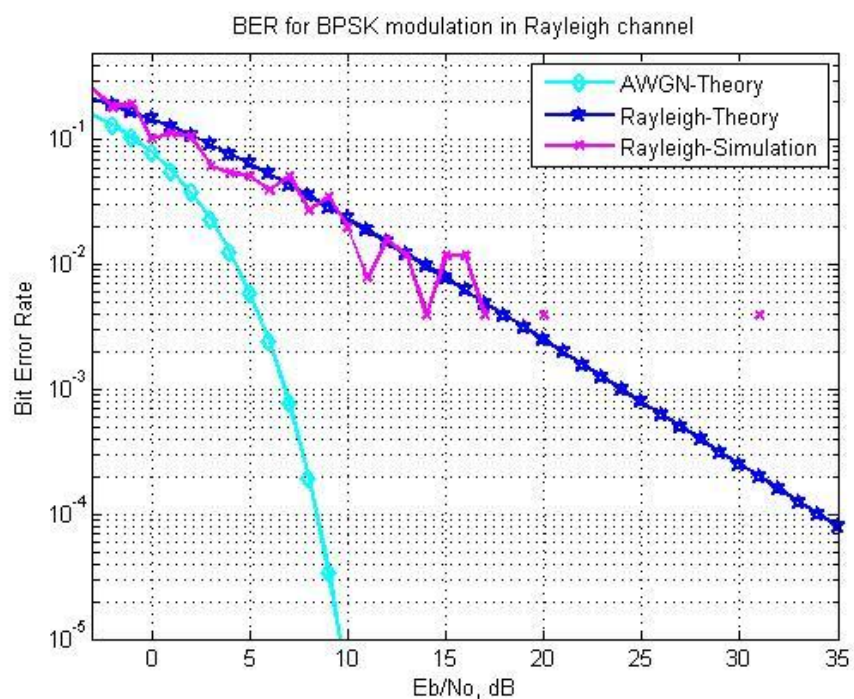

Fig.2 BER for BPSK

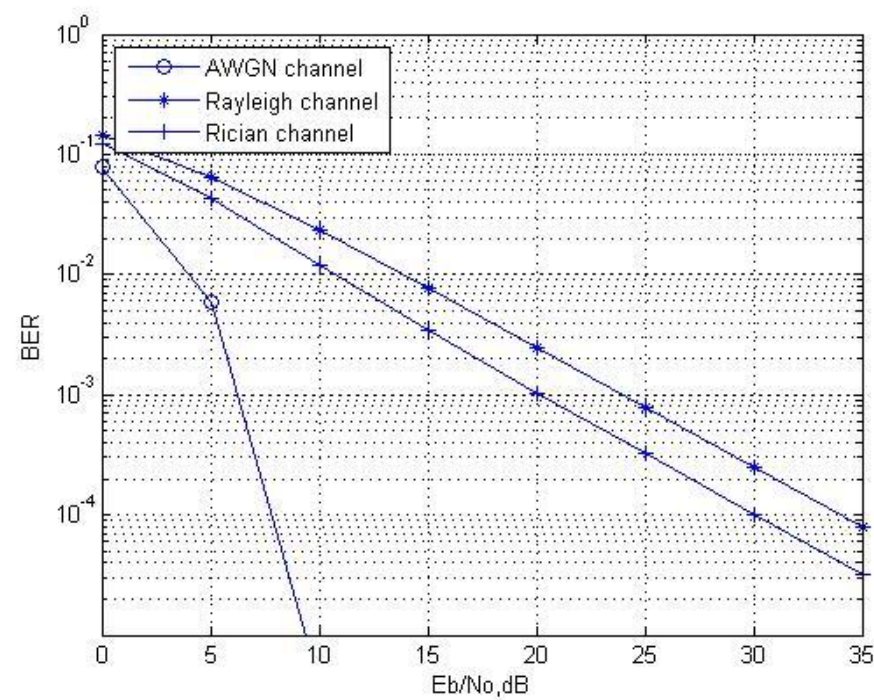

Fig. 3 BER for $\mathrm{K}=2$

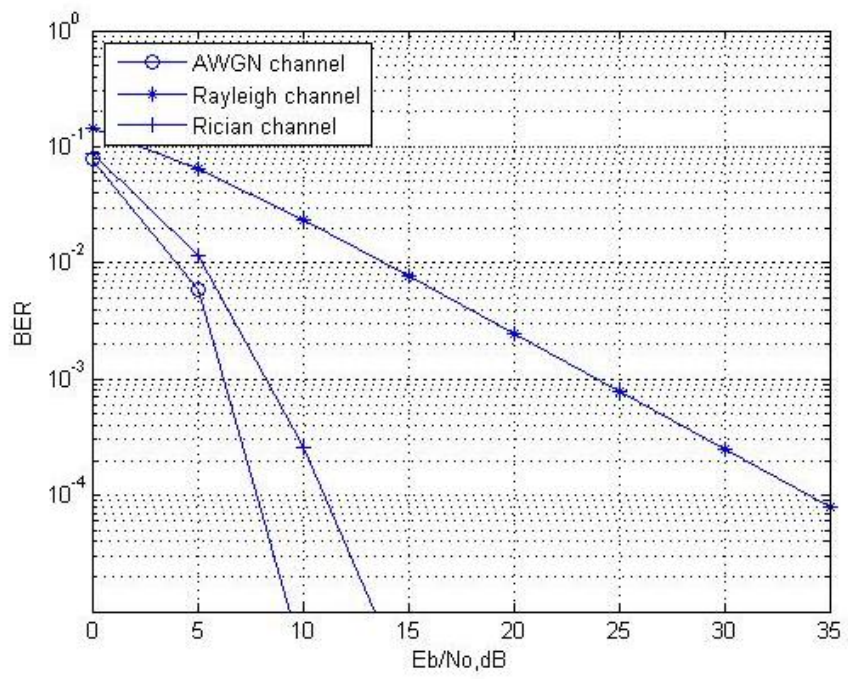

Fig.4 BER with $\mathrm{K}=15$ 
UGC Approved Journal

IJIREEICE

International Journal of Innovative Research in Electrical, Electronics, Instrumentation and Control Engineering

ISO 3297:2007 Certified

Vol. 5, Issue 7, July 2017

\section{CONCLUSION}

For better communication in future MIMO-OFDM technique is proposed. In addition to that, Markov chain technique is used for independently data transmission. Markov Chain uses separate and independent channels. Each channel has its separate error rate which helps user to transmit data independently. Thus it helps to carry out errorless data transmission.

\section{REFERENCES}

[1]. Ertugrul basar, senior member, "On multiple-input multiple-output ofdm with Index modulation for next generation wireless Networks", IEEE transactions on signal processing, IEEE, 2016.

[2]. Preben Mogensen, Kari Pajukoski , Esa Tiirola, Eeva Lähetkangas, Jaakko Vihriälä, Seppo Vesterinen , Matti Laitila, Gilberto Berardinelli, Gustavo W. O. Da Costa, Luis G. U. Garcia,Fernando M. L. Tavares, Andrea F. Cattoni, "5G small cell optimized radio design",Globecom workshop,2013.

[3]. Rui Wang, Honglin Hu, Senior Member, IEEE and Xiumei Yang, "Potentials and Challenges of C-RAN Supporting Multi-RATs towards 5G Mobile Networks", IEEE ACCESS, 2014.

[4]. Yuki Arikawa, Kenji Kawai, Hiroyuki Uzawa, and Satoshi Shigematsu, "Practical Resource Scheduling in Massive-cell Deployment for 5G Mobile Communications Systems", International Symposium on Intelligent Signal Processing and Communication Systems, 2015.

[5]. Sánchez-Salas, D.A. Cuevas-Ruíz, J.L, "N-states Channel Model using Markov Chains", Fourth Congress of Electronics, Robotics and Automotive Mechanics, 2007 